\title{
Intra- and interindividual variation in the power law exponent for area summation
}

\author{
J. O. RAMSAY \\ McGill University, Montreal, Quebec H3A IBI, Canada
}

\begin{abstract}
Subjects were required to indicate the magnitudes of subjective sums of areas of regular 20 -sided polygons by three methods-generation of a polygon of matching area, estimation of the ratio of the subjective sum to a fixed area, and magnitude estimation. Power law exponents were estimated for each subject by maximum likelihood estimation. A very large amount of interindividual exponent variation was observed. Exponents resulting from mag. nitude estimation were consistent on the average with previous results, but exponents from area matching and ratio estimation were much larger and close to unity. When subjects were given feedback on the discrepancy between their responses and physical area summation, they readily shifted their exponents toward unity.
\end{abstract}

A number of factors are known to affect the size of the exponent in the power law for a particular pairing of stimulus and response continua, and the investigation of these influences has been a central strategy in research into its significance. The bulk of this literature describes experiments in which exponents are estimated by aggregating data across individuals. Although individual differences in exponents have been appreciated for some time (Dawson \& Mirando, 1975; Engeland \& Dawson, 1974; Rule \& Markley, 1971; Wanschura \& Dawson, 1974), they have received comparatively less attention. The purpose of this paper is to explore exponent variation by an experimental technique and statistical analysis particularly well suited to within-subject studies.

As well as demonstrating intersubject variation in the exponent with a new vividness, these studies were also designed to show a measure of cognitive control over responses in a simple psychophysical task which is reflected directly in the size of the exponent. These results suggest that what have been classically thought of as sensory phenomena also involve higher level processes.

One of the problems in the investigation of the power law exponent is to separate response effects from stimulus effects. A number of authors have claimed a need to employ two exponents, one for the stimulus modality and one for the response modality

This work was supported by Natural Sciences and Engineering Research Council of Canada Grant APA 320. The author would like to acknowledge the valuable assistance of Susan Scanlon and Justine Sergent. Correspondence and reprint requests should be addressed to the author at Department of Psychology, McGill University, 1205 Docteur Penfield Avenue, Montreal, Quebec H3A 1BA, Canada. A documented computer program for obtaining the maximum likelihood estimates of the parameters of the models used in this paper is also available from the author.
(Attneave, 1962; Curtis, Attneave, \& Harrington, 1968; Curtis \& Fox, 1969; Rule \& Curtis, 1973, 1977; Rule, Curtis, \& Markley, 1970; Curtis \& Rule, Note 1). That is, if a response, $r_{i}$, is recorded to a stimulus whose measured value is $a_{i}$, the psychophysical model is

$$
r_{i}^{z}=k a_{i}{ }^{y} .
$$

Since it is not possible to estimate $\mathrm{z}$ and $\mathrm{y}$ separately in the conventional magnitude estimation paradigm, it has been implicitly assumed either that $z=1$ or that it is $y / z$ that is being estimated. However, if the subject is asked to estimate the subjective sum or difference of two stimuli, it is possible to compute separate estimates using the model

$$
r_{i j}^{z}=k\left(a_{i}^{y} \pm a_{j}^{y}\right),
$$

where $r_{i j}$ designates the response to stimuli $i$ and $j$. Rule and Curtis (1977) related the two issues of exponent variability and the two-stage model in a study of area and numerousness using magnitude estimation of differences. Employing within-subject exponent estimates, they discovered a substantial correlation across modalities in output exponent $z$ and no correlation for input exponent y. However, they also reported large intersubject variation in both exponents for both modalities.

In this paper, the need for a two-exponent model is accepted. However, the additional complexity introduced by this model is offset by introducing a task (area matching) that eliminates the need to use a separate response exponent explicitly. The first experiment applies this paradigm, while the following two experiments employ a ratio estimation and magnitude estimation of area sums for comparison purposes. The final experiment employs the area 
matching technique to show a form of cognitive control over the size of the exponent.

\section{MATCHING AREAS TO SUMS OF AREAS}

In this experiment, subjects were required to choose an area whose subjective magnitude matched the subjective sum of the other areas. This paradigm permits the direct investigation of subjective summability without the complication introduced when the response must be transformed differently from the stimulus. A similar experiment with line lengths was carried out by Krueger (1970).

\section{Method}

The 12 subjects were undergraduate and graduate students at McGill University. They were seated one at a time about $1 \mathrm{~m}$ from a television screen, On each trial, three geometrically similar regular 20-sided polygons appeared on the screen. The stimulus polygons appeared side by side in the lower half of the screen and the response polygon was centered in the top half. The diameter of the response polygon could be changed in linear relationship with the angle of rotation of a knob. The stimulus polygons changed from trial to trial and 100 trials were generated by the ordered pairs of 10 polygons ranging in area from 2.4 to $24 \mathrm{~cm}^{2}$ in equal steps. The order of presentation was randomized uniquely for each experimental session. The display was generated and the results recorded by a PDP-11 computer. Subjects were given a practice series of nine trials.

Although no fixed set of instructions was used, the subjects were told to set the area of the variable polygon equal (as opposed to apparently equal) to the sum of the areas of the two fixed polygons on each trial. At the conclusion of each trial, the subject had to push a button to record the result of that trial, and had to turn the knob to the position producing the largest possible response polygon before the next set of polygons would appear. This was intended to reduce sequential response effects. The largest possible area of the adjustable polygon was $174 \mathrm{~cm}^{2}$, and this considerably exceeded the largest response of any subject.

Analysis. Since the analysis of the data was novel in some respects, it requires some discussion. Each set of 100 trials was analyzed using the following model:

$$
a_{i j}{ }^{z}=p a_{i}^{y}+q a_{j}^{y}+t+c_{1} T_{i j}+c_{2} I_{i j}+e_{i j} \text {. }
$$

In this equation, $\mathrm{a}_{\mathrm{ij}}$ is the physical area of the response polygon produced upon presentation of polygons with areas $a_{i}$ and $a_{j}$ on the left and right, respectively. Parameters $p$ and $q$ are regression coefficients for the stimulus area, $t$ is a constant term, and $c_{1}$ and $c_{2}$ are regression coefficients for two types of bias. The quantity $T_{i j}$ is the trial number on which areas $a_{i}$ and $a_{j}$ were presented less 50.5 , and $\mathrm{I}_{\mathrm{ij}}$ is an indicator variable having the value of +1 if the larger area is on the left, 0 if the areas are equal, and -1 if the larger area is on the right. Thus, $c_{1}$ is a measure of trial number bias and $c_{2}$ of position bias or the extent to which $a_{i j}$ differs from $a_{j i}$.

From the standpoint of fitting the parameters to the model, it is important to note that position bias, constant bias, and $\mathrm{T}_{\mathrm{ij}}$ are mutually stochastically independent, and that the remaining departures from the strictly additive model are both mutually dependent and dependent on the three linear bias coefficients $t, c_{1}$, and $c_{2}$ in varying degrees. For example, the hypothesis $\mathrm{z} \neq \mathrm{y}$ is so highly correlated with the hypothesis that $\mathrm{p}=\mathrm{q} \neq 1$ that these are not worth entertaining at the same time.

Thus, this general model permits the following departures from perfect subjective summability: (1) $z \neq y$, (2) $p=q \neq 1$, (3) $p \neq q$,
(4) $t \neq 0$, (5) $c_{1} \neq 0$, and $(6) c_{2} \neq 0$. In fitting the model, the significant linear effects 4,5 , and 6 were first included, and then the effects 1,2 , and 3 were assessed conditionally on these.

In fitting the model, it was assumed that $\mathrm{a}_{\mathrm{ij}}{ }^{\mathrm{z}}$ was distributed about the right side of Equation 3 with a lognormal distribution. This is equivalent to saying that the logarithm of $a_{i j}{ }^{2}$ is normally distributed about the logarithm of the right side of Equation 3 and is consistent with the usual practice of fitting straight lines by least squares in the logarithms of the stimulus and response measures in the conventional magnitude estimation paradigm. The unknown parameters in the model were estimated by maximum likelihood, and the $\log$ likelihood of the actual responses $a_{i j}$ is given by

$$
\log L=N \log (2 / \sigma)-\left(2 \sigma^{2}\right)^{-1} \sum_{i} \sum_{j}\left(\log a_{i j}{ }^{2}-\log a_{i j}{ }^{*}\right)^{2},
$$

where $a_{i j}{ }^{*}$ is the right side of Equation 3. Numerical maximization of this function with respect to the parameters to be estimated yieids maximum likelihood estimates. The contribution of each of the possible departures from subjective summability was assessed by the asymptotic likelihood ratio test (Beck \& Arnold, 1977), and confidence intervals for exponent y were computed using the usual procedure for computing asymptotic confidence intervals for maximum likelihood estimates. The sample sizes $(\mathrm{N}=100)$ in these analyses are large enough to justify such asymptotic techniques.

The dispersion parameter $\sigma^{2}$ requires special comment. Its maximum likelihood estimate is

$$
s^{2}=N^{-1} \sum_{i} \sum_{j}\left(\log a_{i j}^{2}-\log a_{i j}^{*}\right)^{2},
$$

which is simply the variance of estimate in the log scale. Although seldom reported in experimental studies, it conveys very important information because of its invariance with respect to changes in scale in the independent variable $a$ in the case of $z=y$. The quantity $\sigma / z$ is also invariant with changes in $z$. Essentially, this ratio indicates response variability when the effects of the stimuli $a_{i}$ and $a_{j}$ are partialed out, and hence indicates the overall difficulty of the task. Estimates of $\sigma / z$ can be compared across a wide variety of experimental situations where lognormal fitting is used. Further discussion can be found in Ramsay (1977).

\section{Results and Discussion}

The most striking result (see Table 1) is the large interindividual variation in exponent $y$. The standard deviation of $y$ is .41 , and this is about 15 times as large as the mean standard error of estimate for $y$. The average value for $y$ is $1.17 \pm .20$, and this value is very discrepant from the values typically found in magnitude estimation experiments with area and from Dawson's (1971) value of .58 in his subjective summation experiment.

The majority of subjects showed some type of bias or departure from a simple summation model. The presence of substantial constant bias is consistent with the response model of Irwin and Corballis (1968) and McGill (1960), but could also be said to arise from a sensory threshold effect such as that proposed by Ekman (1958). Trial bias may be due to a shift in the subjective summation strategy over trials. Although a separate exponent, $z$, for the response area was computed, in no instance was it found to differ significantly from the stimulus $y$ and is therefore not reported. 
Table 1

Exponent, Relative Standard Error, and Sources of Bias for Matching Areas and Sums of Areas

\begin{tabular}{|c|c|c|c|c|c|c|c|}
\hline \multirow[b]{2}{*}{ Subject } & \multicolumn{2}{|c|}{ Exponent y } & \multirow[b]{2}{*}{$\sigma / y$} & \multirow[b]{2}{*}{ Position $\left(c_{2}\right)$} & \multicolumn{2}{|c|}{ Source of Bias } & \multirow[b]{2}{*}{$\mathrm{p}=\mathrm{q} \neq 1$} \\
\hline & Mean & SE & & & Constant (t) & Trial $\left(c_{1}\right)$ & \\
\hline H.O.B. & .74 & .02 & .20 & n.s. & $*$ & $* *$ & $*$ \\
\hline J.C.G. & .76 & .02 & .12 & n.s. & n.s. & $* *$ & n.s. \\
\hline D.O.B. & .85 & .02 & .16 & $*$ & $* *$ & $* *$ & n.s. \\
\hline F.P.F. & .86 & .03 & .13 & n.s. & n.s. & n.s. & n.s. \\
\hline C.A.B. & .98 & .07 & .11 & n.s. & $* *$ & n.s. & n.s. \\
\hline R.L.C. & .98 & .04 & .13 & n.s. & n.s. & n.s. & n.s. \\
\hline A.O.B. & 1.02 & .05 & .11 & $* *$ & $*$ & $* *$ & n.s. \\
\hline S.M.S. & 1.06 & .03 & .07 & n.s. & n.s. & n.s. & $*$ \\
\hline D.L.M. & 1.43 & .06 & .09 & $*$ & n.s. & $*$ & n.s. \\
\hline A.B.W. & 1.68 & .09 & .07 & n.s. & n.s. & n.s. & n.s. \\
\hline S.W.M. & 1.82 & .04 & .12 & n.s. & $* *$ & $*$ & $*$ \\
\hline G.T.P. & 1.84 & .18 & .10 & n.s. & $*$ & n.s. & n.s. \\
\hline Mean & 1.17 & .05 & .12 & & & & \\
\hline
\end{tabular}

Note-SE is one-half the width of the $95 \%$ confidence interval around exponent $y$. n.s. $=p>.05 . \quad{ }^{*} p \leqslant .05 . \quad{ }^{* *} p \leqslant .001$.

In attempting to explain the surprisingly large average value of the exponent, it could be argued that the upper limit on the range of response inevitable with any use of physical response apparatus had an effect. While the response in this experiment did not approach the largest possible setting, and there was no evidence of a ceiling effect in the residuals, it is possible that subjects adjusted their overall response strategy in order to avoid the response ceiling. This would result in an underestimation of the subjective sum, and, in turn, in an inflated estimate of the exponent. The next experiment was designed in part to answer this objection.

On the other hand, average exponents from studies such as Dawson's (1971) are a result of employing magnitude estimation. Memory plays a stronger role in this paradigm because the response on the first trial is arbitrary and provides a standard which must somehow be remembered and employed on subsequent trials. This second experiment was also designed to eliminate the memory load of the magnitude estimation task.

\section{RATIO ESTIMATION OF SUBJECTIVE SUMS OF AREAS}

\section{Method}

Ten undergraduate and graduate students participated in this experiment, including one (S.M.S.) who had participated in the previous experiment. The subjects were required to estimate the ratio of the sum of the areas of the two stimulus polygons to that of the upper polygon, which in this experiment was fixed at $19.6 \mathrm{~cm}^{2}$.

For these data, the model was

$$
r_{i j}^{z}=\left(a_{i}^{y}+a_{j}^{y}\right) / a_{0}{ }^{y}+t+c_{1} T_{i j}+c_{2} I_{i j}+e_{i j} \text {, }
$$

where $a_{0}$ is the area of the fixed polygon. The regression coefficients $p$ and $q$, used in Equation 3, were eliminated because of the very high correlation between their estimates and that of response exponent $z$. The latter is of more importance in this experiment, and the hypothesis $z=1$ was of particular interest.

\section{Results and Discussion}

Table 2 contains the results which are, on the whole, similar to those in the previous experiment. Again, significant constant and trial biases were common, while position bias was not detected. The range of individual exponents is again striking. Four of the response exponents were significantly different from unity, but with no predominating direction and with a mean of 1.00 .

The average stimulus exponent in this experiment was 1.14 , as compared with 1.17 in the previous experiment, leaving us more confident that the large average exponent was not due to response ceiling effects. This tends to focus attention on the possibility that it is the memory load in the magnitude estimation experiment that led to Dawson's (1971) finding. The next experiment is essentially a replication of that experiment.

Table 2

Exponents, Relative Standard Error, and Sources of Bias for Ratio Estimates of Sums of Areas

\begin{tabular}{|c|c|c|c|c|c|c|}
\hline \multirow[b]{3}{*}{ Subject } & \multirow{2}{*}{\multicolumn{2}{|c|}{ Exponent y }} & \multirow[b]{3}{*}{ Exponent $z$} & \multicolumn{3}{|c|}{ Source of Bias } \\
\hline & & & & \multirow[b]{2}{*}{$\sigma / z$} & \multirow{2}{*}{$\begin{array}{l}\text { Con- } \\
\text { stant }\end{array}$} & \multirow[b]{2}{*}{ Trial } \\
\hline & Mean & SE & & & & \\
\hline K.N.C. & .60 & .02 & $.80^{* *}$ & .13 & n.s. & $* *$ \\
\hline L.F.I. & .68 & .03 & 1.02 & .13 & n.s. & $* *$ \\
\hline M.L.P & .73 & .02 & 1.03 & .10 & n.s. & n.s. \\
\hline S.C.H. & .80 & .03 & $1.08^{*}$ & .11 & n.s. & $*$ \\
\hline J.A.F. & 1.04 & .03 & $1.18 * *$ & .10 & n.s. & n.s. \\
\hline J.D.C. & 1.14 & .03 & .93 & .14 & n.s. & * \\
\hline B.A.F. & 1.40 & .05 & .90 & .18 & $*$ & $* *$ \\
\hline S.M.S. & 1.61 & .03 & $1.14^{*}$ & .12 & $*$ & $*$ \\
\hline R.L.S. & 1.62 & .06 & .92 & .21 & $*$ & n.s. \\
\hline F.G.E. & 1.75 & .09 & .96 & .19 & $* *$ & $* *$ \\
\hline Mean & 1.14 & .04 & 1.00 & .14 & & \\
\hline
\end{tabular}

Note-SE is one-half the width of the $95 \%$ confidence interval around exponent $y$. All contributions from position bias $\left(c_{2}\right)$ were nonsignificant. n.s. $=p>.05 . \quad{ }^{*} p \leqslant .05 . \quad{ }^{*} p \leqslant .001$. 


\section{MAGNITUDE ESTIMATION OF SUBJECTIVE SUMS OF AREAS}

\section{Method and Analysis}

On each trial, each of nine subjects was presented with two polygons side by side and centered in the screen. He was asked to assign any number he wished to the subjective sum of the first pair of areas and to assign numbers to subsequent pairs so that the numbers were proportional to their subjective sums. These instructions, as in the previous two experiments, made reference to "areas" rather than to "apparent areas." Nine subjects were involved. Other details were as in Experiment 1.

The model for these data was

$$
r_{i j}{ }^{2}=b\left(a_{i}^{y}+a_{j}^{y}\right)+c_{1}{ }^{T}{ }_{i j}+c_{2}{ }_{i j}+e_{i j} .
$$

The regression coefficient $b$ had of necessity to be estimated for every special case of the model. This coefficient was correlated so highly with a constant bias that the latter was omitted from this model. Also, the strong interaction between exponents $y$ and $z$ and this regression coefficient made it relatively difficult to detect cases where $z \neq 1$.

\section{Results and Discussion}

The results are displayed in Table 3 . Since a significant departure from the hypothesis $z=1$ was detected in only two cases, the values of $y$ and $\sigma$ are a consequence of setting $\mathrm{z}$ to unity. In this experiment, the average value of .71 for the exponent is much more in line with previous results, and in particular is not significantly different from the values of .6 found by Stevens and Guirao (1963), .58 found by Dawson (1971), or the value of .81 for areas of polygons found by $M$. Teghtsoonian (1965). On the other hand, the differences between this mean value and those of the exponents in Experiments 1 and 2 are significant at the .01 level.

Experiment 1 raised two main questions: Why was the average exponent so discrepant with previously reported results, and why was there so much intersubject variability in exponent estimates? The results

Table 3

Exponents, Relative Standard Error, and Sources of Bias for Magnitude Estimates of Sums of Areas

\begin{tabular}{lccccc}
\hline & \multicolumn{2}{c}{ Exponent y } & & & Trial \\
Subject & Mean & SE & $\begin{array}{c}\text { Expo- } \\
\text { nent z }\end{array}$ & $\sigma / 2$ & Bias \\
\hline F.J.K. & .38 & .04 & 1.00 & .16 & n.s. \\
D.B. & .49 & .04 & 1.46 & .11 & $* *$ \\
S.E.D. & .70 & .05 & 1.57 & .13 & $* *$ \\
A.L.M. & .71 & .05 & 1.25 & .07 & $* *$ \\
C.B.M. & .72 & .05 & $.52^{* *}$ & .12 & $*$ \\
M.J.W. & .78 & .05 & $1.56^{*}$ & .17 & $* *$ \\
T.C. & .80 & .04 & 1.05 & .13 & n.s. \\
R.Z. & .90 & .05 & .83 & .12 & $*$ \\
H.R. & .95 & .04 & .81 & .20 & n.s. \\
Mean & .71 & 05 & 1.12 & .13 & \\
\hline
\end{tabular}

Note-SE is one-half the width of the $95 \%$ confidence interval around exponent $y$. All but one contribution from position bias $\left(c_{2}\right)$ were nonsignificant. n. $5 .=p>.05 \quad{ }^{*} p \leqslant .05 .{ }^{* *} p \leqslant .001$. of Experiments 2 and 3 illuminate the first problem in that they indicate that there is something about the magnitude estimation task that yields a smaller average exponent. As suggested above, this result may be related to the need to recall at each trial the response given on the previous trial or on the first trial.

The question of intersubject variability raises the issue, among others, of whether subjects adopt a response strategy in a task that is reflected in their exponent values. In all three studies, some subjects reported that they were aware that areas of circles increase at a faster rate than their diameters, and that this influenced their responses. M. Teghtsoonian (1965) found that the exponent for areas of circles differed according to whether subjects were required to judge "how large they are, in terms of area," or "how big they look." It may be, then, that the exponent produced by a particular subject is a reflection of his conscious attempt to achieve a target of performance conveyed to him by the instructions. If so, one would expect that feedback on that performance would modify his exponent in a direction predictable by the nature of the feedback. The next experiment attempts to investigate this.

\section{EFFECT OF FEEDBACK ON AREA MATCHING}

\section{Method}

The 20 McGill undergraduate and graduate students who participated in this study did not participate in the previous studies. They were randomly assigned to an experimental and a control group, each of size 10.

The task on each trial was identical to that in Experiment 1 except that a subject received two blocks of 49 trials, each block being generated by the ordered pairs of seven polygons ranging in area from 3.4 to $24.0 \mathrm{~cm}^{2}$ in equal steps.

Each subject in the experimental group was told at the end of the first block of trials whether his response polygons tended to have area greater or less than the actual sums, but without specifying the degree of error. The difference between the average response area and the average sum of stimulus areas determined the feedback. The subjects were then asked to do the experiment a second time, but without a specific request to adjust their responses to more closely match the physical summation. The control subjects were asked to repeat the experiment without any comment on their previous performance.

\section{Results and Discussion}

The exponents obtained for each subject and block for the simplified model,

$$
a_{i j}{ }^{y}=a_{i}{ }^{y}+a_{j}{ }^{y}+e_{i j},
$$

are displayed in Table 4 . The difference between the average exponents of the two groups for the first block of trials is not significant, and neither is the difference between average exponents within the control group between the two blocks. For the control 
Table 4

Exponents for Two Blocks of Trials for Experimental and Control Groups

\begin{tabular}{cccccc}
\hline & \multicolumn{2}{c}{ Experimental Group } & & \multicolumn{2}{c}{ Control Group } \\
\cline { 2 - 3 } Subject & Block 1 & Block 2 & & Block 1 & Block 2 \\
\hline 1 & .51 & $.84^{* *}$ & & .50 & $.46^{* *}$ \\
2 & .67 & $.85^{* *}$ & & .50 & $.55^{* *}$ \\
3 & .68 & $.84^{* *}$ & .51 & $.65^{* *}$ \\
4 & .73 & $1.01^{* *}$ & .62 & $.74^{* *}$ \\
5 & .82 & $.91^{*}$ & .68 & $.65 \dagger$ \\
6 & .92 & $1.08^{* *}$ & .80 & $.77^{\dagger}$ \\
7 & .98 & $1.24^{* *}$ & .80 & $.85^{*}$ \\
8 & 1.02 & $.98^{* *}$ & & 1.04 & $1.06 \dagger$ \\
9 & 1.19 & $.91^{* *}$ & 1.15 & $1.26 \dagger$ \\
10 & 1.45 & $1.28^{* *}$ & & 1.28 & $1.64^{*}$ \\
Mean & .90 & .99 & .79 & .86 \\
SD & .28 & .16 & .28 & .36 \\
\hline
\end{tabular}

${ }^{*} p<.05$ for the degree of shift in exponent from first to second block. $\quad * * p<.001$ for the degree of shift in exponent from first to second block. $\quad t p \geqslant .05$ for the degree of shift in exponent from first to second block.

group, the correlation between the exponents for the two blocks of trials is 960 , indicating a high degree of test-retest reliability for the exponent. Nevertheless, six control group subjects showed a significant shift in exponent on the basis of a within-subject likelihood ratio test.

The degree of shift towards unity was measured by the quantity $\left(y_{1}-y_{2}\right) \operatorname{sgn}\left(y_{1}-1\right)$; that is, the difference between the first and second exponents with the sign of this difference changed if the initial exponent was less than one. All experimental group subjects showed a positive shift towards unity, while only three control group subjects did. The difference between the average shifts toward unity yields $t=4.1$ $(p=.001)$. Another index of the effect of feedback is the change in the standard deviation of the exponents. As Table 4 indicates, the standard deviation decreased substantially in the experimental group while it increased in the control group.

There were no significant differences between blocks or groups in the estimates of $\sigma / y$. For the control group, the correlation between these estimates for the two blocks was .71 $(\mathrm{p}<.05)$.

\section{CONCLUSIONS}

Although it was thought at one time that the power law exponent was a relatively stable index of sensory processes which varied significantly only with stimulus modality, contemporary accounts have had to accommodate a variety of additional factors. These include the effect of range ( $R$. Teghtsoonian, 1971, 1973) and various effects of response modality (Stevens, 1971, and the cited literature on the twostage model). A comparison of the results of Experiment 3 with those of Experiments 1 and 2 indicates that the nature of the task also has an effect, with magnitude estimation exponents being substantially smaller than those produced by area matching or ratio production.

Even when stimulus range was held constant, and response modality and task effects explicitly allowed for, as they were in this study, there remains a large amount of intra- and intersubject variation. Experiment 4 suggests that a subject's exponent is under substantial cognitive or conscious control, and casual reports show that subjects can often verbalize an explicit response strategy. The conclusion which emerges is that the power law exponent is an index not only of sensory processes, but also of higher order cognitive events comparable in subtlety and complexity to those involved in more traditional problem-solving tasks. Thus, what has traditionally been thought of as a purely psychophysical problem in reality raises questions about higher order information processing. It would seem that the classical notion of psychophysics as the study of sensation independent of a subject's ability to learn and to choose among more complex strategies for responding to stimulation has been too narrowly conceived.

\section{REFERENCE NOTE}

1. Curtis, W. W., \& Rule, S. J. Evidence for a two-stage model of magnitude estimation. Paper presented at the Mathematical Psychology Meeting, La Jolla, California, 1972.

\section{REFERENCES}

Attneave, F. Perception and related areas. In S. Koch (Ed.), Psychology: A study of a science (Vol. 4). New York: McGrawHill, 1962.

Beck, J. V., \& ARnold, K. J. Parameter estimation in engineering and science. New York: Wiley, 1977.

Curtis, D. W., Attneave, F., \& Harrington, T. L. A test of a two-stage model of magnitude judgment. Perception \& Psychophysics, 1968, 3, 25-31.

Curtis, D. W., \& Fox, B. E. Direct quantitative judgments of sums and a two-stage model of psychophysical judgments. Perception \& Psychophysics, 1969, 5, 89-93.

Dawson, W. E. Magnitude estimation of apparent sums and differences. Perception \& Psychophysics, 1971, 9, 368-374.

Dawson, W. E., \& Mirando, M. A. Sensory-modality opinion scales for individual subjects. Perception \& Psychophysics, 1975, 17, 596-600.

Ekman, G. Two generalized ratio scaling methods. Journal of Psychology, 1958, 45, 287-295.

Engeland, W., \& Dawson, W. E. Individual differences in power functions for a 1 -week intersession interval. Perception \& Psychophysics, 1974, 15, 349-352.

Irwin, R. J., \& Corballis, M. C. On the general form of Stevens' law for loudness and softness. Perception \& Psychophysics, 1968, 3, 137-143.

Krueger, L. E. Apparent combined length of two-line and four-line sets. Perception \& Psychophysics, 1970, 8, 210-214.

McGiLL, W. J. The slope of the loudness function: A puzzle. In H. Gulliksen \& S. Messick (Eds.), Psychological scaling: Theory and applications. New York: Wiley, 1960.

Ramsay, J. O. Maximum likelihood estimation in multidimensional scaling. Psychometrika, 1977, 42, 241-266. 
Rule, S. J., \& Curtis, D. W. Reevaluation of two models for judgments of perceptual intervals. Perception \& Psychophysics, $1973,14,433-436$.

Rule, S. J., \& Curtis, D. W. Subject differences in input and output transformations from magnitude estimation of differences. Acta Psychologica, 1977, 41, 61-65.

Rule, S. J., Curtis, D. W., \& Markley, R. P. Input and output transformations from magnitude estimation. Journal of Experimental Psychology, 1970, 86, 343-349.

Rule, S. J., \& Markley, R. P. Subject differences in crossmodality matching. Perception \& Psychophysics, 1971, 9, 115-117.

Stevens, S. S. Issues in psychophysical measurement. Psychological Review, 1971, 78, 426-450.

Stevens, S. S., \& Guirao, M. Subjective scaling of length and area and the matching of length to loudness and brightness. Journal of Experimental Psychology, 1963, 66, 177-186.
Teghtsoonian, M. The judgment of size. American Journal of Psychology, 1965, 78, 392-402.

Teghtsoonian, R. On the exponents in Stevens' law and the constant in Ekman's law. Psychological Review, 1971, 78, $71-80$.

TEGHTSOONIAN, R. Range effects in psychophysical scaling and a revision of Stevens' law. American Journal of Psychology, 1973, 86, 3-27.

Wanschura, R. G., \& Dawson, W. E. Regression effect and individual power functions over sessions. Journal of Experimental Psychology, 1974, 102, 806-812.

(Received for publication August 28, 1978; revision accepted September 17, 1979.) 